\title{
Profilaxis de tuberculosis en niños y adultos sometidos a trasplante de órganos sólidos y precursores hematopoyéticos
}

\author{
Mónica Lafourcade
}

\section{Prophylaxis against tuberculosis in pediatric and adult patients undergoing solid organ and hematopoietic stem cells transplantation}

Recipients of SOT and HSCT constitute a risk group for becoming ill with tuberculosis (TB). The prevalence of active TB in patients undergoing TOS is higher than in patients undergoing HSCT, probably for the shortest period of immunosuppression of the latter. Most TB cases occur in transplant patients by reactivation of latent infection after immunosuppression, which occurs most often within the first year post-transplant, causing graft loss and in some cases death. Relevant variables to assess the risk of TB infection in a transplant recipient are the medical history of donor and recipient, images, microbiology and tuberculin tests and interferon gamma levels. PPD is routinely performed in the donor and in the recipient before transplantation. If PPD is $>5 \mathrm{~mm}$ in the recipient or $>10 \mathrm{~mm}$ in the donor, it is neccesary to exclude active TB (pulmonary and renal) (A2). It is recommended chemoprophylaxis in recipients PPD $(+)$ and in recipients with recent seroconversion (B3), if the donor has a history of untreated TB, there was contact to someone with active TB (B3), the radiological imeges are suspect (A2) and interferon gamma release assays is (+) (B2). The selected drug is isoniazid (C3).

Key words: Tuberculosis, prophylaxis, transplant, solid organ transplantation, hematopoietic stem cells transplantation.

Palabras clave: Tuberculosis, profilaxis, trasplante, trasplante de órganos sólidos, trasplante de precursores hematopoyéticos.

\section{Introducción}

L os pacientes receptores de trasplantes constituyen un grupo de riesgo para enfermar de tuberculosis (TBC) y enfrentan grandes dilemas diagnósticos y terapéuticos; su presentación clínica es atípica y la sensibilidad de las técnicas de diagnóstico disponibles es baja. Por otro lado, los fármacos anti TBC poseen alta toxicidad e interacciones con los antineoplásicos, lo que dificulta el manejo de esta enfermedad.

La mayoría de los casos de TBC en pacientes sometidos a trasplantes ocurren por reactivación de una infección latente luego de la inmunosupresión. Existen casos documentados de infección a través del injerto ${ }^{1}$ y adquisición nosocomial ${ }^{2}$ pero son excepcionales.

La enfermedad, en términos generales, se produce con más frecuencia dentro del primer año post-trasplante, causando pérdida del injerto y en algunos casos la muerte. En trasplante de órganos sólidos (TOS), dos tercios de los casos de TBC activa se presentan en el primer año posttrasplante y es común su manifestación extra pulmonar y diseminada (30-50\% de los casos) ${ }^{3}$.

\section{Epidemiología}

En las últimas décadas, los programas de prevención de TBC en países desarrollados han logrado disminuir su incidencia; no obstante, los países en vías de desarrollo aun mantienen cifras elevadas ${ }^{4}$. En Chile, la tasa de incidencia de TBC en la población general ha descendido drásticamente en los últimos años, desde $77 / 100.000$ habitantes en el año 1980 a 14/100.000 habitantes en el 2008, con diferencias regionales importantes, siendo la más elevada en la Región de Arica-Parinacota con 35/100.0005. A los factores de riesgo clásicamente descritos para infección por Mycobacterium tuberculosis, se han sumado la coinfección por virus de inmunodeficiencia humana (VIH) y el trasplante. Se estima que la prevalencia de TBC activa en pacientes sometidos a TOS en la mayoría de los países desarrollados es de 1,2 a $6,4 \%$, mientras que en zonas de alta endemia, ésta puede llegar hasta $15 \%{ }^{6}$. La incidencia varía según el órgano trasplantado, siendo más alta en trasplante pulmonar comparado con otros órganos ${ }^{5}$. En trasplante de precursores hematopoyéticos (TPH), ésta es menor que en TOS, probablemente debido al menor tiempo de inmunosupresión al que son sometidos los pacientes. En estudios norteamericanos, la cifras fluctúan entre 0,6 y 1\%; sin embargo, en países donde la endemia es mayor, la incidencia es más elevada: 1,6\% en España, $5 \%$ en Hong Kong y Taiwán ${ }^{7}$.

\section{Factores de riesgo}

Trasplante de órganos sólidos. Se han definido pocos factores de riesgo con claridad para el desarrollo de TBC
Laboratorio de Microbiología Clínica Santa María, Santiago.

La autora declara no tener conflictos de interés.

Correspondencia a: mlafourcade@hotmail.com 
en estos pacientes. La principal dificultad para obtener datos precisos está dada por la calidad de los estudios donde la mayoría son retrospectivos y series de casos. Además, la información disponible se refiere fundamentalmente a trasplante renal y por tanto, no es necesariamente aplicable a los receptores de otros trasplantes ${ }^{8-10}$.

Dentro de los factores de riesgo descritos para el desarrollo de TBC están la terapia con OKT3 o anticuerpos anti-linfocitarios ${ }^{6}$, diabetes mellitus, enfermedad hepática crónica, co-infecciones (CMV, micosis profunda, neumonía por Pneumocystis jiroveci y Nocardia sp) ${ }^{11}$, edad avanzada y el uso de ciclosporina. Otros factores de riesgo tienen que ver con antecedentes de exposición a TBC, PPD positivo ( $\geq$ a $5 \mathrm{~mm}$ ) o imágenes pulmonares compatibles $^{10}$.

Trasplante de precursores hematopoyéticos. En este grupo los factores de riesgo descritos son EICH crónica, trasplante alogénico e irradiación corporal total ${ }^{12}$.

\section{Evaluación de candidatos a trasplante}

Para evaluar el riesgo de infección tuberculosa de un receptor, se debe recolectar exhaustivamente su historial médico, incluyendo actividad laboral, viajes, antecedente de contacto previo con $M$. tuberculosis, si existió tratamiento anti-tuberculoso y duración de éste.

La utilidad de la prueba de tuberculina en la evaluación de un candidato a trasplante es controversial. Esta puede estar falsamente negativa en 50 a $80 \%$ de ellos por el estado de anergia en que se encuentran y también puede haber falsos positivos por interferencia con la vacunación con BCG (Bacillus Calmette Guerin); sin embargo, el efecto absoluto de ésta se pierde si la vacunación se realizó hace más de 10 años ${ }^{13}$. Las guías de la Sociedad Americana de Trasplante recomiendan el empleo de tuberculina en forma independiente del estado de vacunación del candidato ${ }^{14}$.

Respecto del empleo de IGRAS (Interferon Gamma Release Assays), la sensibilidad y especificidad de estos métodos varía según el tipo empleado y la población estudiada y fluctúa entre 50 a $100 \%$ y 85 a $100 \%$, respectivamente, en los diferentes estudios ${ }^{15}$. Hay pocos reportes de detección de $\mathrm{TBC}$ latente con estos métodos, por lo que se requiere una mejor evaluación. Un estudio en que se comparó Quantiferon ${ }^{\circledR}$ TB gold con test de tuberculina para el diagnóstico de TBC latente en candidatos a trasplante hepático, demostró que ambas técnicas eran comparables ${ }^{16}$.

Un paciente con test de tuberculina positivo, imágenes pulmonares sugerentes de TBC activa o cicatrizada (le- siones infiltrativas, cavitarias o fibronodulares apicales, nódulos calcificados, patología pleural) o con sintomatología sospechosa, debe someterse a una búsqueda activa del bacilo de Koch, a través de cultivo y amplificación genética en muestras de expectoración, lavado bronquioalveolar y orina ${ }^{17}$.

Toda enfermedad por M. tuberculosis en el donante o receptor debe ser tratada hasta su regresión microbiológica y resolución radiológica antes de considerar el trasplante ${ }^{17}$. Un donante con TBC activa o sospecha de ella no debe ser considerado para trasplante ${ }^{1,18}$.

\section{Tratamiento de la tuberculosis latente}

El tratamiento de TBC latente supone un riesgo variable de toxicidad, principalmente hepática y requiere un control muy estricto de los niveles plasmáticos de la terapia inmunosupresora. A la fecha, isoniazida es el fármaco de elección para la profilaxis y ha demostrado ser efectiva en trasplante renal, hepático y cardíaco ${ }^{19-21}$. La ATS y el CDC recomiendan nueve meses de terapia. Algunos autores sugieren que el riesgo de hepatotoxicidad de la isoniazida sobrepasa el beneficio de su uso. No obstante, el seguimiento de la función hepática cada 15 días ha demostrado ser una estrategia útil para controlarla.

Recomendación Nº 8: Quimioprofilaxis de tuberculosis en candidatos a TOS y TPH

- Realizar PPD en el donante y el receptor. Si el PPD es $\geq$ de $5 \mathrm{~mm}$ en el receptor o > de $10 \mathrm{~mm}$ en el donante, se debe evaluar una $\mathrm{TBC}$ activa (pulmonar y renal) (A2)

- Una TBC activa en el donante o receptor debe ser tratada antes de considerar el trasplante (C3)

- Se recomienda efectuar quimioprofilaxis en el receptor PPD (+) y/o seroconversión reciente (B3), si el donante tiene el antecedente de TBC no tratada, existió contacto con persona con TBC activa (B3), las imágenes radiológicas son sospechosas (A2) e IGRAs (+) (B2)

- Se recomienda efectuar quimioprofilaxis con isoniacida durante nueve meses, diariamente o dos veces por semana (C3). En adultos $5 \mathrm{mg} / \mathrm{kg} /$ día y en niños 10-15 mg/kg/día. Agregar piridoxina (vitamina $B_{6}$ ) 25-50 mg/día para disminuir los riesgos de neurotoxicidad por fármacos (C3 ). Alternativamente, si se presenta intolerancia a isoniacida, se recomienda emplear la asociación de etambutol más moxifloxacina o levofloxacina( C3)

Agradecimientos: Un especial agredecimiento a Victorino Farga por sus oportunos consejos científicos y editoriales. Un maestro, ejemplo de dedicación y sencillez. 


\section{Resumen}

Los pacientes receptores de trasplante constituyen un grupo de riesgo para enfermar de tuberculosis (TBC). La prevalencia de TBC activa en pacientes sometidos a TOS es mayor que en TPH, probablemente por el menor tiempo de inmunosupresión. La mayoría de los casos de TBC en pacientes trasplantados ocurren por reactivación de infección latente luego de la inmunosupresión, la que se produce con más frecuencia dentro del primer año posttrasplante, causando pérdida del injerto y en algunos casos la muerte. Para evaluar el riesgo de infección tuberculosa de un receptor son relevantes elementos tales como el historial médico del donante y receptor, las imágenes, el estudio microbiológico y pruebas como la tuberculina y ensayos que miden interferón gamma. De rutina se realiza PPD en el donante y el receptor previo al trasplante. $\mathrm{Si}$ el PPD es $\geq$ de $5 \mathrm{~mm}$ en el receptor o $\geq$ de $10 \mathrm{~mm}$ en el donante, se debe descartar una TBC activa (pulmonar y renal) (A2). Se recomienda efectuar quimioprofilaxis en el receptor PPD $(+)$ y seroconversión reciente (B3), si el donante tiene el antecedente de TBC no tratada, existió contacto con persona con TBC activa (B3), las imágenes radiológicas son sospechosas (A2) e IGRAs (+) (B2). El medicamento de elección para la profilaxis es isoniacida (C3).

\section{Referencias bibliográficas}

1.- Peters T G, Reiter C G, Boswell R L. Transmission of tuberculosis by kidney transplantation. Transplantation 1984; 38: 514-6.

2.- Jereb J A, Burwen D R, Dooley S W, Haas W H, Crawford J T, Geiter L J, et al. Nosocomial outbreak of tuberculosis in a renal transplant unit: application of a new technique for restriction fragment length polymorphism analysis of Mycobacterium tuberculosis isolates. J Infect Dis 1993; 168: 1219-24.

3.- Singh N, Paterson D L. Mycobacterium tuberculosis infection in solid-organ transplant recipients: impact and implications for management. Clin Infect Dis 1998; 27: 1266-77.

4.- Farga V. Tuberculosis. Tercera ed: Mediterráneo; 2011.

5.- Torre-Cisneros J, Doblas A, Aguado J M, San Juan R, Blanes M, Montejo M, et al. Tuberculosis after solid-organ transplant: incidence, risk factors, and clinical characteristics in the RESITRA (Spanish Network of Infection in Transplantation) cohort. Clin Infect Dis 2009; 48: 1657-65.

6.- Muñoz P, Rodríguez C, Bouza E. Mycobacterium tuberculosis infection in recipients of solid organ transplants. Clin Infect Dis 2005; 40: 581-7.

7.- Cordonnier C, Martino R, Trabasso P, Held T K, Akan H, Ward M S, et al. Mycobacterial infection: a difficult and late diagnosis in stem cell transplant recipients. Clin Infect Dis 2004; 38: 1229-36.
8.- Basiri A, Moghaddam SM, Simforoosh N, Einollahi B, Hosseini M, Foirouzan A, et al. Preliminary report of a nationwide case-control study for identifying risk factors of tuberculosis following renal transplantation. Transplant Proc 2005; 37: 3041-4.

9.- $\quad$ Torres J, Aguado J M, San Juan R, Andrés A, Sierra P, López-Medrano F, et al. Hepatitis C virus, an important risk factor for tuberculosis in immunocompromised: experience with kidney transplantation. Transpl Int 2008; 21: 873-8.

10.- John G T, Shankar V, Abraham A M, Mukundan U, Thomas PP, Jacob C K. Risk factors for post-transplant tuberculosis. Kidney Intern 2001; 60: 1148-53.

11.- Tharayil John G, Shankar V, Talaulikar G, Mathews M S, Abraham Abraham M, Punnakuzhathil Thomas P, et al. Epidemiology of systemic mycoses among renal-transplant recipients in India. Transplantation 2003; 75 : 1544-51.

12.- Mary M S, Yuen K Y, Woo P C, Luk W K, Tsang K W, Lam W K, et al. Risk factors for pulmonary tuberculosis in bone marrow transplant recipients. Am J Resp Crit Care Med 1998; 158: 1173-7.

13.- Farhat M, Greenaway C, Pai M, Menzies D. False-positive tuberculin skin tests: what is the absolute effect of BCG and non-tuberculous mycobacteria? Int J Tuberc Lung Dis 2006; 10: 1192-204.

14.- Society A T. Targeted tuberculin testing and treatment of latent tuberculosis infection. Am J Resp Crit Care Med 2000; 161 (Suppl): 221-47.
15.- CDC. Updated Guidelines for Using Interferon Gamma Release Assays to Detect Mycobacterium tuberculosis Infection-United States, 2010. MMWR Morbid Mortal Wkly Rep 2010; 59.

16.- Manuel O, Humar A, Preiksaitis J, Doucette K, Shokoples S, Peleg AY, et al. Comparison of quantiferon-TB gold with tuberculin skin test for detecting latent tuberculosis infection prior to liver transplantation. Am J Transplant 2007; 7: 2797-801.

17.- Fischer S A, Avery R K, Practice ASTIDCo. Screening of donor and recipient prior to solid organ transplantation. Am J Transplant 2009; 9 Suppl 4: S7-18.

18.- Mourad G, Soulillou J P, Chong G, Pouliquen M, Hourmant M, Mion C. Transmission of Mycobacterium tuberculosis with renal allografts. Nephron 1985; 41: 82-5.

19.- Agarwal S A, Gupta S, Dash S C, Bhowmik D, Tiwari S C. Prospective randomised trial of isoniazid prophylaxis in renal transplant recipient. Intern Urol Nephrol 2004; 36: 425-31.

20.- Benito N, Sued O, Moreno A, Horcajada J P, González J, Navasa M, et al. Diagnosis and treatment of latent tuberculosis infection in liver transplant recipients in an endemic area. Transplantation 2002; 74: 1381-6.

21.- John GT, Thomas P P, Thomas M, Jeyaseelan L, Jacob C K, Shastry J C. A double-blind randomized controlled trial of primary isoniazid prophylaxis in dialysis and transplant patients. Transplantation 1994; 57 : 1683-4. 